\title{
Preliminary evidence for cue-induced alcohol craving modulated by serotonin transporter gene polymorphism
} rs1042173

\section{Nassima Ait-Daoud ${ }^{1}$, Chamindi Seneviratne ${ }^{1}$, Justin B. Smith ${ }^{1}$, John D. Roache ${ }^{2}$, Michael A. Dawes $^{2}$, Lei Liu ${ }^{3}$, Xin-Qun Wang ${ }^{3}$ and Bankole A. Johnson'}

${ }^{1}$ Department of Psychiatry and Neurobehavioral Sciences, University of Virginia, Charlottesville, VA, USA

2 Department of Psychiatry, University of Texas Health Science Center at San Antonio, San Antonio, TX, USA

${ }^{3}$ Department of Public Health Sciences, University of Virginia, Charlottesville, VA, USA

\section{Edited by:}

Lorenzo Leggio, Catholic University of

Rome, Italy

\section{Reviewed by:}

Giovanni Addolorato, Catholic University of Rome, Italy

George Kenna, Brown University, USA

\section{*Correspondence}

Nassima Ait-Daoud, Department of Psychiatry and Neurobehavioral Sciences, University of Virginia, P.O. Box 800623, Charlottesville, VA 22908-0623, USA.

e-mail: nat7b@virginia.edu
We previously have shown that cue-induced alcohol craving and propensity for higher drinking are modulated by allelic differences in SLC6A4 associated with serotonin transporter (5-HTT) expression level alterations. In an independent study, we characterized another polymorphism, SNP rs1042173, in 3'-untranslated region (3'-UTR) of the same gene, which also altered 5-HTT expression levels; the T allele of rs1042173 was associated with lower mRNA and protein levels. In subsequent analyses, the TT genotype was found to be associated with higher drinking intensity in alcohol-dependent (AD) individuals of Caucasian descent. Building upon these findings, we hypothesized that the low-expressing TT genotype associated with intense drinking would predict higher craving for alcohol in AD individuals. In this pilot study, we sought to test our hypothesis by examining $34 \mathrm{His}-$ panic AD volunteers (mean age, 34.8 years) for rs 1042173 genotype-based [i.e., TT versus TG/GG (Gx)] differences in subjective response to alcohol. We employed a human laboratory paradigm and analyzed the data using a linear mixed-effects model (SAS $®$ PROC MIXED) to assess treatment, cue procedures, and genotype main effects as well as the two-way interaction effects between them. On subjective "urge to drink" and "crave for a drink," we found a significant main effect of the cue experiment $(p \leq 0.01)$ and an interaction effect between genotype and cue effects $(p<0.05)$. TT genotype was associated with higher urge to drink $(p=0.002)$ and crave for a drink $(p=0.005)$ when exposed to alcohol cue. Our results not only support the hypothesis that rs1042173 is a genetic marker for cue-induced alcohol craving among $A D$ males but also are suggestive of a neurobiological mechanism associated with the rs1042173-TT genotype that triggers a disproportionate craving in response to alcohol consumption, which in turn may lead to more intense drinking. Future studies with larger sample sizes are needed to characterize the interactive effects of the serotonin transporter-linked polymorphic region (5'-HTTLPR)-L-allele reported in our previous study and of the rs1042173-TT genotype on cue-induced alcohol craving.

Keywords: alcohol, craving, serotonin transporter, SLC6A4 gene, polymorphism, rs1042173, genotype

\section{INTRODUCTION}

Serotonin pathways are important modulators of the reward system in the brain both in natural pleasurable activities such as feeding and sexual activity and in addictive behavior. Serotonincontaining neurons project diffusely to various areas known to be involved in the reward system, including the nucleus accumbens, ventral tegmental area, substantia nigra, hippocampus, amygdala, and prefrontal cortex (Hayes and Greenshaw, 2011). The precise mechanisms by which serotonin modulates the reward system are still being elucidated. Nearly all of the serotonin receptor subtypes have been linked to areas in the brain associated with addiction and reward.

The presynaptic serotonin transporter (5-HTT) has been investigated extensively over the years in the addiction genetics field as an important regulator of drinking behavior (Heinz et al., 2000). A wealth of literature exists on genetic associations between 5-HTT genetic polymorphisms and phenotypes of alcoholism; among these, the most widely studied polymorphism is the 5HTT $5^{\prime}$-regulatory promoter region variant, the 5-HTT-linked polymorphic region [5'-HTTLPR; long (L) and short (S) alleles]. Recently, Seneviratne et al. (2009) reported another allelic variation at the 5-HTT gene (SLC6A4) $3^{\prime}$-untranslated region $\left(3^{\prime}\right.$ UTR) single-nucleotide polymorphism (T/G), rs1042173, altering 5-HTT mRNA and protein levels; the T allele-transfected HeLa cells had significantly lower mRNA and protein levels than the G-allele-transfected cells. In the same study, in a group of alcoholdependent $(\mathrm{AD})$, treatment-seeking individuals who were participating in an outpatient clinical trial, the authors showed that TT 
genotype, compared with the TG/GG genotypes, was associated with significantly higher drinking intensity in Caucasian individuals (Seneviratne et al., 2009). The authors also detected a similar, albeit a non-significant, trend in Hispanic individuals and speculated that the higher drinking intensity associated with the TT genotype may have been due to lower levels of 5-HTT expression resulting in higher synaptic serotonin concentrations, which in turn may lead to hazardous drinking behaviors (Seneviratne et al., 2009). In a subsequent pharmacogenetic study, also conducted by our group, we have shown that individuals of both Caucasian and Hispanic descent carrying LL and TT genotypes showed a reduction in their drinking severity compared with the non-carriers when treated with an anti-serotonergic drug (Johnson et al., 2011). Taken together, these previous findings suggest a modulatory effect of rs1042173 on the subjective response to alcohol in individuals of both Caucasian and Hispanic descent.

In the present study, we utilized a human laboratory paradigm to test whether Hispanic individuals who were homozygous for the T allele, compared with G-allele carriers, would display stronger cue-induced craving for alcohol. Human laboratory paradigms offer a unique advantage over an outpatient setting in that they effectively minimize the influence of various other environmental factors on a behavioral phenotype, such as subjective response to alcohol and propensity to drink, thereby refining the genetic effects on the phenotype (Kaplan et al., 1985; Walitzer and Sher, 1990; Ait-Daoud et al., 2009).

Additionally, we conducted an acute tryptophan depletion test, which currently represents the most established human challenge test to manipulate the central serotonergic system in vivo and assess the behavioral effects of reduced serotonin metabolism in the brain. The tryptophan depletion test also allows us to examine whether acute tryptophan depletion (Delgado et al., 1991; Young and Leyton, 2002; Neumeister, 2003) would alter the cue-induced propensity to drink among $\mathrm{AD}$ individuals with the TT, TG, and GG genotypes.

\section{MATERIALS AND METHODS SUBJECTS}

A total of 34 Hispanic male AD individuals (aged between 21 and 50 years) were enrolled into the study. All subjects were in good health as determined by a complete physical examination, electrocardiogram, and laboratory screening tests within an acceptable range. Female participants had to have a negative pregnancy test at intake and be willing to use an acceptable form of contraception, which included an oral contraceptive pill, barriers and spermicide, or hormonal implants. To be eligible, subjects had to be actively drinking $\geq 21$ standard drinks per week for males and $\geq 14$ standard drinks per week for females during the 30 days prior to enrollment. Informed consent was obtained from all subjects.

Exclusion criteria included a current axis I Diagnostic and Statistical Manual of Mental Disorders, 4th edition (DSM-IV; American Psychiatric Association, 1994) diagnosis of mental illness except for nicotine or alcohol dependence, as determined by mental status and psychiatric interview using the Structured Clinical Interview for DSM-IV (First et al., 1994), and a lifetime history of major depression, panic disorder, bipolar disorder, eating disorder, or thought disorder. Other exclusion criteria included a current history of significant medical illness as determined by history, complete physical examination, and electrocardiogram, a positive family history of major depression (in the parents), and those currently seeking treatment for alcohol dependence.

\section{DESIGN}

The study was designed to test the original hypothesis of the role of $5^{\prime}$-HTTLPR polymorphisms (LL, LS, or SS) on cue-induced alcohol craving [see Ait-Daoud et al. (2009) for a detailed description of the study design]. Briefly, subjects were randomized using an urn procedure into balanced groups of LL, LS, and SS individuals of similar age and drinking level. Each participant had four test sessions, with each session separated by at least $24 \mathrm{~h}$ to allow for tryptophan reserves to be replenished. On the morning of each testing day, participants were asked to consume the tryptophan-containing (Tryp+) or tryptophan-depleted (Tryp-) beverage. Objective and subjective measures of craving were collected at scheduled intervals throughout the experiment: three times before cue exposure ( $\mathrm{t} 1-\mathrm{t} 3$ ), just after cue exposure ( $\mathrm{t} 4$ ), and at the end of the experiment $(\mathrm{t} 5)$, which corresponds to about an hour after cue exposure. Exposure to the cues took place $5.5 \mathrm{~h}$ after consumption of the Tryp + or Tryp- beverage to allow sufficient time for alterations in tryptophan availability to occur (Delgado et al., 1990, 1994).

\section{TRYPTOPHAN DEPLETION METHOD}

A detailed description of the tryptophan depletion procedure has been given in the original publication (Ait-Daoud et al., 2009). To summarize, tryptophan depletion was applied in a doubleblind, counterbalanced, within-subject design. Tryptophan depletion was achieved through a tryptophan-deficient amino acid mixture administered $5 \mathrm{~h}$ prior to testing. The Tryp- beverage contained large amino acids that compete with carrier-mediated transport and uptake of tryptophan into the brain. The Tryp+ beverage or sham drink contained a mixture of cornstarch and grapefruit concentrate.

\section{End of experiments}

At the end of each experimental session, participants were provided with a meal rich in proteins that included tryptophan.

\section{CUE CRAVING METHOD}

\section{Alcohol cue exposure procedure}

A detailed description of the alcohol cue exposure appeared in the original publication (Ait-Daoud et al., 2009). To summarize, alcoholic drink preference (i.e., beer, liquor, or wine) was established at screening. At the time of cue exposure, participants were seated comfortably in a private room and presented with their alcoholic drink of choice along with a medicine cup containing $2 \mathrm{ml}$ of the drink. Participants were instructed to pick up the can or bottle, open it, pour a small amount into a glass, and hold the glass against their chin to smell the drink. They were then instructed to take the contents of the medicine cup and swirl it around their mouth before swallowing. Subjective measures of craving were collected during $\mathrm{t} 1 \mathrm{-} \mathrm{t} 5$. At the end of the session, participants were allowed to consume the drink to avoid habituation and extinction of responses to the craving cues. The alcohol cue exposure task took approximately $5 \mathrm{~min}$ to perform. 


\section{Neutral cue exposure procedure}

Here participants were asked to choose between iced tea and lemonade during screening.

\section{CRAVING INSTRUMENTS}

\section{Subjective measures}

Visual analog scales (VAS) of alcohol craving were adapted from the Tiffany Craving Questionnaire. The visual analog scales of interest included 100-mm lines with adjectival descriptions of "I have an urge for a drink" and "I crave a drink right now" that were anchored on the left by "not at all" and on the right by "extremely."

\section{GENOTYPING FOR rs1042173 POLYMORPHISMS}

All DNA samples were collected at baseline for the purpose of genotyping $5^{\prime}$-HTTLPR polymorphisms to randomize participants for the human laboratory experiment described above. The alleles of rs 1042173 were genotyped with pre-made $\operatorname{TaqMan}^{\circledR}$ genotyping assays (Applied Biosystems, Foster City, CA, USA) as reported previously (Seneviratne et al., 2009).

\section{ETHICAL APPROVAL}

Ethical approval was provided by the institutional review board at the University of Texas Health Science Center at San Antonio.

\section{STATISTICAL ANALYSIS}

The general timeline for the assessments was $\mathrm{t} 1-\mathrm{t} 3$ (pre-cue), $\mathrm{t} 4$ (cue), t5 (post-cue). VAS questionnaires (i.e., "I have an urge for a drink" and "I crave a drink right now") were administered at all time points described above for the nine different sessions. The VAS measures were described as their mean and SE or $95 \%$ confidence interval (CI). For the VAS questionnaires, we selected two outcomes: the difference between time points $\mathrm{t} 3$ and $\mathrm{t} 4$ and the area under the curve of time points $\mathrm{t} 3-\mathrm{t} 5$ inclusive. In our analysis, the profiles of the questions of interest were examined first to ensure use of the appropriate statistical model. Then, a linear mixedeffects model (SAS ${ }^{\circledR}$ PROC MIXED; SAS Institute Inc., Cary, NC, USA) was used to study the effects of treatment, genotype, and cue as well as the two-way interaction effects between them on the two outcomes for the VAS questionnaires. The analytical model was adjusted for covariates that included the participant's chronological age, average drinking level during the 90 days prior to enrollment, and alcohol severity prior to enrollment as measured on the subscales of severity and frequency of withdrawal and delirium tremens on the Addiction Severity Index. Due to the small sample size, Satterthwaite approximation was used for calculating the number of degrees of freedom. Two-way interactions of treatment (Tryp+ or Tryp-), genotype, and cue were first included in the models but were excluded from the final model if not significant. The significance level of all tests was specified at 0.05 , and all tests were two-sided. All analyses were carried out in $\mathrm{SAS}^{\circledR}$ version 9.2 (SAS Institute Inc.).

\section{RESULTS}

Twenty-three of the 34 participants were G-allele carriers (i.e., TG/GG), and 9 had the TT genotype. For two participants, the genotype of interest was not identified; therefore, they were not included in the analysis. All participants were male. The mean \pm SD chronological age was 34.9 (10.0) for TG/GG and 34.7
(4.6) for TT; age of onset of problem drinking was 22.0 (6.9) for TG/GG and 17.8 (2.3) for TT; baseline drinking levels were 8.5 (4.7) in the TG/GG group and 9.6 (7.5) in the TT group.

For the duration of the inpatient stay, there was no report of alcohol withdrawal symptoms in any of the participants. All participants tolerated well their drinks, and no one experienced any adverse events. Tryptophan depletion achieved a $71 \%$ reduction in $5.5 \mathrm{~h}$ based on the measurement of free plasma tryptophan level. With 1 day between sessions, tryptophan levels were replenished for the next session, irrespective of whether the preceding session was a tryptophan depletion session or a placebo session, as demonstrated by plasma tryptophan levels that were back to baseline.

For both "urge to drink" and "crave for a drink," there was a significant main effect of cue $(p \leq 0.01)$ and an interaction between rs1042173 and cue (rs1042173*cue) variables $(p<0.05)$. There was no effect of tryptophan depletion on "urge to drink" and "crave for a drink" ( $p>0.05$; Table 1$)$

As expected, the alcohol cue induced a greater "urge to drink" (mean $=11.29 ; 95 \% \mathrm{CI}=3.82-19.76 ; p=0.004)$ and "crave for a drink" (mean $=10.89 ; 95 \% \mathrm{CI}=2.66-19.13 ; p=0.010)$ compared with the neutral cue. Upon subgrouping the study population based on rs1042173 genotypes, we detected a twofold increase in both "urge to drink" and "crave for a drink" in response to alcohol, within TT carriers (Figure 1). In TT carriers, the mean difference between alcohol and neutral cue-induced "urge to drink" was 20.12 (95\% CI: 7.56-32.69; $p=0.002)$ and "crave for a drink" was 20.13 (95\% CI: 6.22-34.04; $p=0.005$ ). In contrast, within the TG/GG group, alcohol cue did not elicit a significantly greater response than the neutral cue. Furthermore, the "urge to drink" under the alcohol cue in TT genotype individuals was also significantly greater than the scores seen in TG/GG carriers (mean difference $=11.25$; 95\% CI: 0.04-22.46; $p=0.049$ ). The difference for "crave for a drink" between the TT and TG/GG groups was not statistically significant, although the magnitude of difference was similar to what we have seen for "urge to drink" between the two genotype groups (mean difference $=11.05 ; 95 \%$ CI: -5.09 to $27.19 ; p=0.174$ ). Thus, it is reasonable to argue that this discrepancy between responses to the two variables (urge and craving) may have resulted from lack of consistency in how the individuals interpreted the term "craving."

Table 1 | Analysis of variance table for the mean difference between time points post-cue (i.e., $t 4)$ and pre-cue (i.e., $t 3$ ).

\begin{tabular}{|c|c|c|c|c|}
\hline \multirow[t]{2}{*}{ Variable } & \multicolumn{2}{|c|}{ "Urge to drink" } & \multicolumn{2}{|c|}{ "Crave for a drink" } \\
\hline & $F$-value & $p$-Value & $F$-value & $p$-Value \\
\hline $\begin{array}{l}\text { Treatment (tryptophan } \\
\text { depletion) }\end{array}$ & 1.66 & 0.201 & 0.01 & 0.914 \\
\hline rs1042173 (TT, TG/GG) & 0.34 & 0.567 & 0.07 & 0.795 \\
\hline Cue & 9.10 & 0.004 & 6.59 & 0.010 \\
\hline rs1042173*cue & 5.58 & 0.021 & 5.01 & 0.028 \\
\hline $\begin{array}{l}\text { Age of onset of } \\
\text { problem drinking }\end{array}$ & 0.72 & 0.407 & 0.12 & 0.728 \\
\hline
\end{tabular}

p-Values $<0.05$ are shown in bold-faced font. 

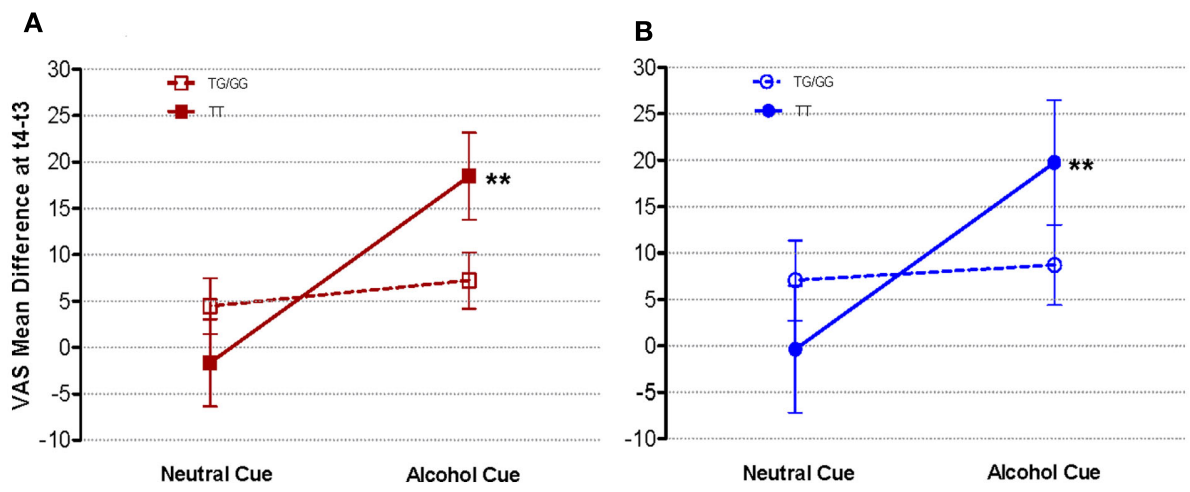

FIGURE 1 | Mean (A) "urge to drink" and (B) "crave for a drink," as a function of rs1042173 genotypes. "Urge to drink" and "crave for a drink" were assessed using visual analog scales (VAS) pre (t3)- and post (t4)-exposure to the alcohol and neutral cues. ${ }^{* *} p<0.01$ for the comparison between the alcohol and neutral cues within the TT genotype group.

There was no effect of tryptophan depletion or age of onset of problem drinking on cue-induced alcohol craving.

\section{DISCUSSION}

The present study confirmed our hypothesis that $\mathrm{AD}$ individuals with the TT genotype, compared with G-allele carriers, would have a higher subjective response to alcohol in Hispanic individuals. Those with the TT genotype, but not G-allele carriers, scored higher on both of the VAS subjective craving measures in the presence of the alcohol cue than in the presence of the neutral cue. That is, when exposed to alcohol, TT carriers, but not the TG/GG carriers, showed significantly greater urge (20.12 points) and craving (20.13 points) for drinking. Urge and craving for drug use are often used synonymously in addiction research literature (Rohsenow and Monti, 1999; Skinner and Aubin, 2010) with a difference being that of intensity. Marlatt (1987) described urge as an "intention to use" and craving as a "temptation to use" drugs, in which case the latter can exist without the former (Tiffany, 1999; Skinner and Aubin, 2010). Considering these factors and practical concerns related to possible inconsistent interpretations among participants, we utilized both "I have an urge for a drink" and "I crave a drink right now" VAS questionnaire items to measure craving. Moreover, in our study, the two questionnaire items were highly correlated with each other $(r=0.930)$, and the TT genotype carriers had higher scores for both items in the presence of the alcohol cue. The word "craving" in the title implies a more inclusive definition, and, therefore, we did not mention both "urge" and "crave" in the title.

These results supplement findings from our previous outpatient and in vitro studies (Seneviratne et al., 2009; Johnson et al., 2011) that showed lower 5-HTT expression levels and more intense alcohol drinking behavior associated with the TT genotype. From a biological and behavioral perspective, it could be argued that lower 5-HTT expression levels associated with the TT genotype may facilitate serotonergic and dopaminergic output in the reward pathways, thereby increasing the desire for more intense drinking, upon exposure to small priming doses. However, in the study conducted by Seneviratne and colleagues, the association between rs1042173 and drinking intensity in Hispanic individuals was not statistically significant although the trend was similar. One possible explanation for this lack of association is that the drinking data were collected retrospectively by self-reports.

Interestingly, in our primary study from the same human laboratory experiments, we reported on another functional polymorphism in SLC6A4 also associated with reduced 5-HTT expression levels in alcoholics (Ait-Daoud et al., 2009). In that study, AD individuals with the LL polymorphism of the $5^{\prime}$-HTTLPR had greater cue-induced alcohol craving and possibly greater propensity for drinking compared with S-allele carriers. These results were further strengthened by a series of biochemical studies that reported higher lifetime drinking associated with lower levels of 5-HT binding and uptake among L-allele carriers (Johnson et al., 2008). In the present study, we were too limited by the relatively small sample size to examine the combined effects of the TT and LL genotypes on alcohol craving; nevertheless, it is noteworthy that in our population, 6 of the 9 TT subjects had the LL genotype; among G-allele carriers, only 6 of 17 possessed the LL genotype.

An important disparity between our primary analysis and the current study is that in the latter we did not find an effect of tryptophan depletion on cue-induced alcohol craving, perhaps because of the smaller sample size in this analysis. We also did not find an effect of age of onset of problem drinking on cue-induced alcohol craving. Classifying alcoholics into clinically meaningful subtypes usually provides better delineation of the psychopathological factors mostly associated with the manifestations and course of the disease. An early age of onset of problem drinking is usually associated with greater alcohol severity and has been used to factor in the role of greater duration of drinking in different types of research settings.

There are several important caveats that need to be considered when evaluating our results. First, this was a relatively small human laboratory study that did not allow us to evaluate the interactive effects between the $5^{\prime}$-HTTLPR and rs1042173 genotypes on cue-induced alcohol craving or the gender differences among "genotype $\times$ treatment" groups. In this context, our study represents a pilot human laboratory investigation that examined the genetic effects of a functional polymorphism on 
cue-induced alcohol craving. Second, because this is a post hoc analysis, subjects were not randomized based on their rs1042173 genotypes; thus, the frequencies of TT versus TG/GG genotypes were not equally distributed, which could have enhanced the observed effects. Third, our experiment was conducted in a very controlled environment and was not designed to measure selfadministered alcohol amounts during the laboratory procedure; this limited our capacity to infer directly the correlations between cue-induced "urge and crave for a drink" and drinking intensity for the same participants. It is noteworthy to mention that we deliberately controlled the drinking level at enrollment to avoid drinking variability between the two groups and to allow participants to safely stay in an inpatient unit without significant negative health consequences.

Despite these limitations, we now have consistently shown that 5-HTT expression levels are associated with the extent of the desire to drink and the severity of drinking. More specifically, we have shown that $\mathrm{AD}$ individuals carrying genetic variants of SLC6A4 that are associated with lower levels of 5-HTT expression are prone to express higher reactivity to alcoholrelated cues. Furthermore, our results highlight cue-induced alcohol craving as a homogeneous behavioral intermediate

\section{REFERENCES}

Ait-Daoud, N., Roache, J. D., Dawes, M. A., Liu, L., Wang, X.-Q., Javors, M. A., Seneviratne, C., and Johnson, B. A. (2009). Can serotonin transporter genotype predict craving in alcoholism? Alcohol. Clin. Exp. Res. 33, 1329-1335.

American Psychiatric Association. (1994). Diagnostic and Statistical Manual of Mental Disorders, 4th Edn. Washington, DC: American Psychiatric Association.

Delgado, P. L., Charney, D. S., Price, L. H., Aghajanian, G. K., Landis, H., and Heninger, G. R. (1990). Serotonin function and the mechanism of antidepressant action: reversal of antidepressant-induced remission by rapid depletion of plasma tryptophan. Arch. Gen. Psychiatry 47, 411-418.

Delgado, P. L., Price, L. H., Miller, H. L., Salomon, R. M., Aghajanian, G. K., Heninger, G. R., and Charney, D. S. (1994). Serotonin and the neurobiology of depression: effects of tryptophan depletion in drug-free depressed patients. Arch. Gen. Psychiatry 51, 865-874.

Delgado, P. L., Price, L. H., Miller, H. L., Salomon, R. M., Licinio, J., Krystal, J. H., Heninger, G. R., and Charney, D. S. (1991). Rapid serotonin depletion as a provocative challenge test for patients with major depression: relevance to antidepressant action and the neurobiology of depression. Psychopharmacol. Bull. 27, 321-330.
First, M. B., Spitzer, R. L., Gibbon, M., and Williams, J. B. W. (1994). Structured Clinical Interview for DSM-IV Axis I Disorders - Patient Edition (SCID-I/P, Version 2.0). New York: Biometrics Research Department, New York State Psychiatric Institute.

Hayes, D. J., and Greenshaw, A. J. (2011). 5-HT receptors and reward-related behaviour: a review. Neurosci. Biobehav. Rev. 35, 1419-1449.

Heinz, A., Jones, D. W., Mazzanti, C., Goldman, D., Ragan, P., Hommer, D., Linnoila, M., and Weinberger, D. R. (2000). A relationship between serotonin transporter genotype and in vivo protein expression and alcohol neurotoxicity. Biol. Psychiatry 47, 643-649.

Johnson, B. A. (2008). Update on neuropharmacological treatments for alcoholism: scientific basis and clinical findings. Biochem. Pharmacol. 75, 34-56.

Johnson, B. A., Ait-Daoud, N., Seneviratne, C., Roache, J. D., Javors, M. A., Wang, X.-Q., Liu, L., Penberthy, J. K., DiClemente, C. C., and Li, M. D. (2011). Pharmacogenetic approach at the serotonin transporter gene as a method of reducing the severity of alcohol drinking. Am. J. Psychiatry 168, 265-275.

Johnson, B. A., Javors, M. A., Roache, J. D., Seneviratne, C., Bergeson, S. E., Ait-Daoud, N., Dawes, M. A., and Ma, J. Z. (2008). Can serotonin transporter genotype predict serotonergic function, chronicity, and

phenotype related to a candidate gene (SLC6A4) and an underlying pathophysiology within the serotonergic system. Medications that curb craving for alcohol have been tested as successful agents for treating alcoholism (Johnson, 2008). Therefore, the present findings will indeed enable us to narrow down the complexity of conducting pharmacogenetic studies by identifying and targeting subpopulations of alcoholics with higher craving.

In summary, we provide evidence of another functional polymorphism in SLC6A4 associated with the subjective response to alcohol in Hispanic AD individuals. Future studies with larger sample sizes are required to characterize better the genetic epistatic effects that contribute to individual variation underlying human drinking behavior.

\section{ACKNOWLEDGMENTS}

We are grateful to the National Institute on Alcohol Abuse and Alcoholism for their generous support through grants 5 K23 AA000329-06 and 5 R01 AA019720-02 awarded to Dr. Nassima Ait-Daoud. We also thank Robert H. Cormier, Jr., for his assistance with manuscript preparation. The authors are entirely responsible for the scientific content of this paper.

severity of drinking? Prog. Neuropsychopharmacol. Biol. Psychiatry 32, 209-216.

Kaplan, R. F., Cooney, N. L., Baker, L. H., Gillespie, R. A., Meyer, R. E., and Pomerleau, O. F. (1985). Reactivity to alcohol-related cues: physiological and subjective responses in alcoholics and nonproblem drinkers. $J$. Stud. Alcohol 46, 267-272.

Marlatt, G. A. (1987). Craving notes. Br. J. Addict. 82, 42-44.

Neumeister, A. (2003). Tryptophan depletion, serotonin, and depression: where do we stand? Psychopharmacol. Bull. 37, 99-115.

Rohsenow, D. J., and Monti, P. M. (1999). Does urge to drink predict relapse after treatment? Alcohol Res. Health 23, 225-232.

Seneviratne, C., Huang, W., Ait-Daoud, N., Li, M. D., and Johnson, B. A. (2009). Characterization of a functional polymorphism in the $3^{\prime}$ UTR of SLC6A4 and its association with drinking intensity. Alcohol. Clin. Exp. Res. 33, 332-339.

Skinner, M. D., and Aubin, H. J. (2010). Craving's place in addiction theory: contributions of the major models. Neurosci. Biobehav. Rev. 34, 606-623.

Tiffany, S. T. (1999). Cognitive concepts of craving. Alcohol Res. Health 23, 215-224.

Walitzer, K. S., and Sher, K. J. (1990). Alcohol cue reactivity and ad lib drinking in young men at risk for alcoholism. Addict. Behav. 15, 29-46.

Young, S. N., and Leyton, M. (2002). The role of serotonin in human mood and social interaction. Insight from altered tryptophan levels. Pharmacol. Biochem. Behav. 71, 857-865.

Conflict of Interest Statement: Professor Bankole A. Johnson has served as a consultant to Johnson \& Johnson (Ortho-McNeil Janssen Scientific Affairs, LLC), Transcept Pharmaceuticals, Inc., D\&A Pharma, Organon, ADial Pharmaceuticals, LLC (with which he also serves as Chairman), Psychological Education Publishing Company (PEPCo), LLC, and Eli Lilly and Company.

Received: 28 October 2011; accepted: 19 January 2012; published online: 16 February 2012.

Citation: Ait-Daoud N, Seneviratne C, Smith JB, Roache JD, Dawes MA, Liu L, Wang X-Q and Johnson BA (2012) Preliminary evidence for cueinduced alcohol craving modulated by serotonin transporter gene polymorphism rs1042173. Front. Psychiatry 3:6. doi: 10.3389/fpsyt.2012.00006

This article was submitted to Frontiers in Addictive Disorders, a specialty of Frontiers in Psychiatry.

Copyright (C) 2012 Ait-Daoud, Seneviratne, Smith, Roache, Dawes, Liu, Wang and Johnson. This is an open-access article distributed under the terms of the Creative Commons Attribution Non Commercial License, which permits noncommercial use, distribution, and reproduction in other forums, provided the original authors and source are credited. 